\title{
Neural tube defects in Gauteng, South Africa: Recurrence risks and associated factors
}

G Teckie, BSc (Hons), MSc (Med), MB BCh, FCP (SA); A Krause, MB BCh, PhD; J G R Kromberg, BA (Soc Work), MA, PhD

Division of Human Genetics, School of Pathology, University of the Witwatersrand and National Health Laboratory Service, Johannesburg, South Africa

Corresponding author: G Teckie (gteckie@hotmail.com)

Background. After congenital heart disease, neural tube defects (NTDs) are the most common serious structural birth defects in human infants. Objectives. To (i) determine the recurrence risks of NTDs in the population of Gauteng; (ii) investigate some of the risk factors shown to be important in the occurrence of NTDs in other populations; and (iii) determine their relative importance in the aetiology of NTDs in the Gauteng population. Methods. A retrospective study was undertaken of 640 families with a member with an NTD. Data were collected from the genetic counselling files held in the Department of Human Genetics for a 28-year period.

Results. A recurrence risk \pm standard deviation (SD) for NTDs of $2.28 \pm 0.9 \%$ (1/45) was calculated for the population. There was no significant difference between the risk of recurrence $0.73 \pm 1.0 \%$ for the black families $(n=98)$ compared with those for the total sample $(N=621)$. The risk rose to $4.16 \%$ after giving birth to two affected children. Analysis of the gender of those with NTDs showed that significantly more female infants (male:female ratio 0.82) were affected. The study also showed that while maternal age was not a significant risk factor for the occurrence of NTDs, maternal parity did play a role, and first and last children were at increased risk. In addition, a higher occurrence of spontaneous abortions and of apparently unrelated congenital malformations in other offspring was found in families with a child with an NTD.

Conclusions. This study provides unique information relevant to the genetic counselling of families with a member with an NTD in our population. All affected families should be referred to a genetics service for appropriate counselling.

S Afr Med J 2013;103(12 Suppl 1):973-977. DOI:10.7196/SAMJ.7119

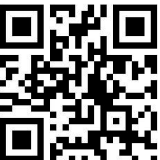

Neural tube defects (NTDs) are a common cause of morbidity and mortality in all ethnic groups in the South African (SA) population, and are reported to occur in about 1 in 800 births. ${ }^{[1]}$ NTDs are generally believed to be multifactorial in aetiology, and a number of genetic and environmental factors have been implicated and shown to be important in their occurrence and recurrence. ${ }^{[2-4]}$ The most important environmental factor is dietary folic acid; since the fortification of staple foods in SA, the number of affected births has declined by about $30 \% \cdot{ }^{[5]}$ Nevertheless, the birth rate is still estimated at 0.98 per 1000 births. $^{[5]}$

\section{Recurrence risks}

In studies performed worldwide, recurrence risks (RRs) of between $2 \%$ and $6 \%$ have been estimated for NTDs, and these risks have been shown to vary with the population incidence of these disorders, the higher risks being found in areas with higher occurrences of 
The authors have been working with Professor Trefor Jenkins for many years. One author (JGRK) was in his department when counselling for families with a child with a neural tube defect (NTD) was first introduced in Johannesburg, and when the first prenatal diagnosis was performed. Another author (AK) trained as a geneticist under the guidance and encouragement of Professor Jenkins. Research into the incidence of NTDs was included in a study on birth defects carried out at Chris Hani Baragwanath Academic Hospital and reported in the SAMJ in 1982. ${ }^{[32]}$ GT, a genetic counselling student in the Department, undertook an MSc research project on NTDs and it produced some unique and informative results. This is the first paper from this project and it presents findings that are relevant to genetic counselling, accurate recurrence risks and an understanding of the risk factors occurring in a South African population.

NTDs. ${ }^{[2,3,6]}$ Where two members of a sibship are affected, the RR for subsequent siblings has been shown to be $2-3$ times as high as that after one affected child. ${ }^{[7,8]}$ No data were available for RRs in SA families with one or more children with an NTD. Such data are required if appropriate risks are to be given in genetic counselling clinics in SA.

\section{Gender}

Multifactorial conditions often show deviations in sex ratios from those of the general population. Most studies have recorded an excess of female births with NTDs, with this finding being more pronounced when anencephalic births were analysed separately. ${ }^{[9,10]}$ Analysis of spina bifida according to the site of lesion showed that lesions occurring in the thoracic region demonstrated a female excess, while those occurring in the lower lumbar and sacral regions were seen more frequently in males. ${ }^{[11]}$

\section{Parental age}

Studies of the effects of maternal age on the occurrence of NTDs have yielded conflicting results. Some studies have shown no maternal age effects, ${ }^{[2,13]}$ some have demonstrated an increased risk in very young mothers and in older mothers, ${ }^{[0]}$ while others suggest that only older mothers have an increased susceptibility. ${ }^{[6]}$ Two large studies failed to identify any paternal age effects on the incidence of NTDs. ${ }^{[6,14]}$

\section{Spontaneous abortions}

A number of studies have documented an increased occurrence of spontaneous abortions in pregnancies of women who have NTDaffected infants. ${ }^{[15-17]}$ The widely accepted explanation for this finding is that many of these spontaneous abortions occur because the fetus is affected by an NTD or other congenital abnormality. It is believed that about half of pregnancies complicated by NTDs will end in spontaneous abortion. ${ }^{[18]}$

\section{Maternal parity}

The effect of maternal parity on the occurrence of NTDs is also unclear. Some studies have shown no significant parity effects, ${ }^{[2,13]}$ while others have demonstrated a higher occurrence in first-born children. ${ }^{[12,14]}$ Buccimazza et al. ${ }^{[9]}$ reported an increased incidence at both extremes of birth order.

\section{NTDs and other malformations}

Estimates of the frequencies of isolated (non-syndromic) congenital malformations in individuals with NTDs vary from around 6\% to
$40 \%$ depending on the methods of assessment and the abnormalities included. ${ }^{[19,20]}$ The malformations most commonly observed were renal and cardiovascular defects, but there was significant variation in anomalies reported by different studies. ${ }^{[1,21,22]}$ Earlier studies of siblings of individuals with NTDs failed to demonstrate an increased risk of other malformations; ${ }^{[212,23]}$ however, more recent studies have found significant increases in the occurrence of apparently unassociated congenital abnormalities in siblings, with a variety of malformations being recorded, particularly in siblings of NTD patients with high spina bifida lesions. ${ }^{[1,24]}$

\section{Objectives}

To investigate (i) the RRs of NTDs; (ii) the sex ratios of affected individuals; (iii) several environmental, genetic and clinical factors including parental age, parity, history of spontaneous abortion; and (iv) the abnormalities associated with NTDs. The collection of such data and its application to the counselling and care of families of patients with NTDs would allow more accurate prediction of RRs and evidence-based prevention strategies.

\section{Methods}

This was a retrospective study of data gathered from the files of patients with a family history of NTDs, counselled by medical geneticists and/ or genetic counsellors at Genetic Counselling Clinics at Chris Hani Baragwanath Academic and Johannesburg hospitals for a 28-year period from 1969 to 1997. Patients with spina bifida occulta or with NTDs which were known to be part of specific genetic syndromes (such as trisomies 13 and 18) were not included in the analysis, as these are considered to be distinct groups with different aetiological factors.

\section{Subjects and patients}

Subjects were identified from the patient records in the Genetic Counselling Clinics at the Division of Human Genetics, University of the Witwatersrand and the National Health Laboratory Service. All patients with diagnoses of NTD, spina bifida, meningomyelocoele, meningocoele, anencephaly or encephalocoele, in themselves or in a family member, together with those who had such a diagnosis in a fetus, were included in this study. Altogether 690 files were identified and, of these, 640 were located and reviewed. Controls for this study were taken from general population statistics where possible. ${ }^{[25]}$ Comparisons were also made with data available for patients seen at the same Genetic Counselling Clinics for unrelated disorders, in this case cystic fibrosis $(n=64)$ and albinism $(n=70)$. Control data on maternal parity were extracted from a study of twins in a similar population by Hain. ${ }^{[26]}$

\section{Data collection}

A checklist was compiled to collect the following data: patient particulars, parental dates of birth, details of the pregnancy (including birth order, teratogen exposure, mode of delivery and outcomes of the pregnancy) where available, a detailed pedigree analysis, history, type of lesion and associated malformations in the affected child, and the occurrence of other abnormalities and pregnancy outcomes in parents of a child with an NTD.

The data were subjected to statistical analysis where necessary and differences between groups were considered significant when $p<0.05$. $\chi^{2}$ analysis was used to determine whether significant differences existed between the experimental and control groups, and Poisson distributions were used to analyse some of the findings on subgroups of data where small numbers were involved.

Ethics approval was granted by the Committee for Research on Human Subjects (Medical) of the University of the Witwatersrand (protocol no. M970125). 


\section{Results}

\section{Recurrence risks}

RRs were calculated from the pedigree information documented in the files and included the results of amniocenteses performed on pregnancies following those affected by NTDs. The number of recurrences for each type of NTD was then compared with the total number of children born to the families to determine the empiric RR for siblings for that type of NTD.

Good family histories spanning at least two generations were available for 621/640 (97\%) families. Twenty-three of these families had two children with NTDs and one family had three affected children (Table 1). There were two families in which pairs of half-siblings were affected. Affected individuals had 1073 unaffected full siblings and 135 unaffected half-siblings. From these observations, an RR \pm standard deviation (SD) of $2.28 \pm 0.9 \%$ was calculated for full siblings. After two affected children, the $\mathrm{RR} \pm \mathrm{SD}$ increased to $4.16 \pm 8.1 \%$. The $\mathrm{RR} \pm \mathrm{SD}$ for half-siblings was $1.46 \pm 2 \%$.

In the 98 black families with individuals with an NTD, there were two recurrences (both in half-siblings) out of 275 pregnancies, giving an $\mathrm{RR} \pm \mathrm{SD}$ of $0.73 \pm 1 \%$, not significantly different from the risk for the whole study group for the sample size available.

RRs were then calculated according to the sex of the proband, and no significant differences $(p>0.05)$ were found after an affected male or an affected female for either spina bifida or anencephaly. Concordance for type of NTD in the recurrence was also examined and a concordance of $94.7 \%$ (18/19 cases) was calculated for families in which the proband had spina bifida, and $75 \%$ (6/8 cases) for families with anencephaly.

Table 1. NTD recurrences in families $(N=621)$ with a member with an NTD

\begin{tabular}{ll}
\hline Siblings & NTD, $\boldsymbol{n}$ \\
\hline Normal/unaffected & \\
Full siblings & 1073 \\
Half-siblings & 135 \\
Total, $N$ & 1208 \\
Affected & \\
Full siblings & 24 \\
1 & 23 \\
2 & 1 \\
Half-siblings & 2 \\
1 & 2 \\
Total, $N$ & 26 \\
Total, $N$ & 1234 \\
NTD $=$ neural tube defect. &
\end{tabular}

\section{Gender}

The gender of the affected individual was available in 619 cases. There were 279 affected males and 340 affected females (male:female ratio (M:F) 0.82) with an excess of female births in both spina bifida and anencephaly (M:F 0.84 and 0.76, respectively). In patients where records of upper and lower spina bifida lesions were available $(n=90)$, upper lesions $(n=34)$ were present in an excess of females (M:F 0.7), while lower lesions $(n=56)$ showed a male excess (M:F 1.15).

\section{Parental age}

Parental age at the birth of affected children was calculated from the dates of birth of parents as recorded in the files and were initially compared with data from the general population. ${ }^{[25]}$ Average maternal age could not be calculated from the 1993 SA Census figures ${ }^{[25]}$ which only documented a range, and was estimated from the files of patients seen at the Genetic Counselling Clinics for cystic fibrosis, in the white population, and albinism, in the black population.

There was no significant difference between the average maternal age at the time of delivery of infants with an NTD (25.6 years; $n=441$ ) compared with mothers of infants with cystic fibrosis $(n=42)$ and mothers of infants with albinism $(n=70)$ (26.1 years for both). Black mothers of infants with NTDs yielded an average maternal age of 26.2 years, which, again, was not significantly different from mothers of children with albinism. There were also no significant differences between the maternal ages of mothers of infants with spina bifida or with anencephaly. The distributions of maternal ages of mothers with NTDs in comparison with mothers in the general population $^{[25]}$ showed similar distributions, with no significant differences between the areas under the two curves $(p>0.05)$.
There was no significant difference between the paternal age distribution of fathers of infants with NTDs ( $n=399$ ) compared with the general population. ${ }^{[25]}$

\section{Spontaneous abortions}

Spontaneous abortions and stillbirths for each nuclear family of an individual with an NTD were recorded. Those known to be due to an NTD, which had already been included in the calculation of the RR for NTDs, were not included in the analysis because it is known that NTD pregnancies are often not carried to term and therefore a significant difference would be expected for this reason alone. The number of miscarriages per couple was compared with that found in the controls (couples with a child with albinism $(n=67)$ or cystic fibrosis $(n=64)$ ), counselled at the Genetic Counselling Clinics. Neither condition is believed to increase the risk of spontaneous abortion, therefore providing suitable controls for this part of the study.

Complete information was available from the files of 550 of the families with affected children with NTDs. There was a significantly increased occurrence of spontaneous abortions in families of individuals affected by NTDs ( $25.1 \%$ of families) compared with the control group (16.\%) (odds ratio 0.64; $95 \%$ confidence interval $0.39-1.05)$. The risk of multiple pregnancy losses $(\geq 3)$ was $3.3 \%$ in the NTD families and $2.2 \%$ in the control families (Table 2).

\section{Maternal parity}

Maternal parity and the birth order of affected children were obtained from the files of 555 families with a member with an NTD. Comparison of these findings to parity of mothers ( $n=2$ 249) sampled randomly from the general population ${ }^{[26]}$ showed that significantly more NTDs occurred in the first pregnancy than in
Table 2. Spontaneous abortions in NTD families and controls

\begin{tabular}{llllllll}
\hline & \multicolumn{7}{c}{ Spontaneous abortions, $\boldsymbol{n}$} \\
\cline { 2 - 8 } Diagnosis & $\mathbf{0}$ & $\mathbf{1}$ & $\mathbf{2}$ & $\mathbf{3}$ & $\mathbf{4}$ & $\mathbf{5 +}$ & Total, $\boldsymbol{N}$ \\
\hline NTD group & & & & & & & \\
$\quad$ Spina bifida & 288 & 63 & 26 & 4 & 4 & 5 & 390 \\
Anencephaly & 124 & 29 & 2 & 3 & 1 & 1 & 160 \\
$\quad$ Total, $N$ & 412 & 92 & 28 & 7 & 5 & 6 & 550 \\
Control group & & & & & & & \\
$\quad$ Albinism & 58 & 7 & 1 & 0 & 1 & 0 & 67 \\
$\quad$ Cystic fibrosis & 52 & 9 & 1 & 1 & 1 & 0 & 64 \\
$\quad$ Total, $N$ & 110 & 16 & 2 & 1 & 2 & 0 & 131 \\
NTD $=$ neural tube defect. & & & & & & &
\end{tabular}


subsequent pregnancies $(43 \%$ v. $22 \%$ in the general population; $p<0.005)$. There was also a significant increase in the occurrence of NTDs in the fifth or higher pregnancies $(p<0.005)$. Findings were similar for spina bifida and anencephaly (Table 3).

\section{NTDs and other malformations}

The occurrence of other non-syndromic malformations not thought to be related to the defect in individuals with NTDs was determined. In addition, an analysis was made of the postulated rate of malformations in siblings of affected individuals. These findings were compared with the occurrence of congenital malformations in the general population as estimated by Harper. ${ }^{[27]}$

Altogether, 56/504 (11\%) of the individuals with NTDs had other isolated abnormalities. The most common were facial clefts (1.2\%), followed by limb malformations and polydactyly. Comparisons of these rates with those in the general population ${ }^{[27]}$ showed that individuals with NTDs had a 3-fold increased risk of having another isolated congenital malformation over the background population risk.

For siblings of patients with NTDs, $4.9 \%$ had an additional malformation, which was equivalent to an approximately $1 / 20(5 \%)$ risk of congenital malformation (other than NTDs). This risk is higher than the $2-3 \%$ risk postulated for the general population. ${ }^{[2]}$

\section{Other factors}

A number of other factors were studied in relation to the occurrence of NTDs in this study. An analysis of exposure to potential teratogens showed that $1.5 \%(9 / 591)$ of the mothers, for whom this information was available, were on antiepileptic therapy at the time of conception, and $0.5 \%(3 / 591)$ of the women had taken traditional medications early in their pregnancies. Six women (1\%) were diabetic patients on insulin, while 34 mothers (5.8\%) of an affected infant reported a flu-like illness or high temperature early in pregnancy, a finding from which it was difficult to draw any conclusions, given the retrospective nature of the study. No significant correlations were demonstrated between the occurrence of NTDs and the season of conception, the occurrence of twins in the families, consanguinity in the families or social class.

\section{Discussion \\ Recurrence risks}

An RR of 2.28\% (1/44) was calculated for the subsequent children of a couple who had one child with an NTD. As expected from the incidence of NTDs in the Gauteng population, which is at the lower end of occurrences of NTDs worldwide ${ }^{[1]}$ the RR also falls at the lower end of the range of risks reported in the literature. For the relatively small sample sizes available, RRs in couples from the black and the white ethnic groups were not significantly different. After giving birth to two affected siblings, the risk of a third affected child was estimated to be $4.16 \%$, almost twice that after one affected child. This observation is probably explained by genetic influences on the aetiology of NTDs and/or repeated or persistent environmental exposures and teratogens. In this study, a small proportion of the mothers had diabetes or they were epileptics on therapy; however, a further study with a larger sample would be required to analyse the impact of various known teratogens on the NTDs in this population. Researchers have postulated that RRs may be underestimated by the fact that families with affected individuals may have fewer children either as a result of increased fetal loss of affected pregnancies or as a result of confounding psychosocial factors. ${ }^{[4]}$

In agreement with other studies, ${ }^{[2,14]}$ we found no difference in the risk of recurrence if the proband was male or female. Further, significant concordance was found for the type of NTD in affected families, a finding which has relevance to genetic counselling for these disorders and has been reported by other authors. ${ }^{[28]}$

Studies suggest that folic acid supplementation may affect not only the occurrence, but also the recurrence of NTDs. ${ }^{[29]}$

Table 3. Birth order of probands in families of NTD patients and in the general population

\begin{tabular}{lllllll}
\hline & \multicolumn{6}{c}{ Birth position of proband, $\boldsymbol{n}$ (\%) } \\
\cline { 2 - 7 } Group & $\mathbf{1}$ & $\mathbf{2}$ & $\mathbf{3}$ & $\mathbf{4}$ & $\mathbf{5 +}$ & Total, $\boldsymbol{N}$ \\
\hline General population $^{[26]}$ & $495(22)$ & $1192(53)$ & $429(19)$ & $106(4.7)$ & $27(1.2)$ & 2249 \\
NTDs & $241(43)$ & $163(29)$ & $90(16)$ & $32(5.7)$ & $29(5.2)$ & 555 \\
$\quad$ Spina bifida & $177(43)$ & $111(28)$ & $64(16)$ & $25(6.2)$ & $20(5)$ & 397 \\
$\quad$ Anencephaly & $64(40)$ & $52(33)$ & $26(16)$ & $7(4.4)$ & $9(5.6)$ & 158 \\
NTD = neural tube defect. & & & & & &
\end{tabular}

At the time of the present study, before the introduction of widespread dietary fortification in our population, ${ }^{[5]}$ the incidence of NTDs was higher and RRs were also probably higher. Anecdotal evidence suggests, ${ }^{[30]}$ and further research is needed to confirm, that there has been an appreciable decrease in recurrence as well as in occurrence risks with the introduction of folic acid fortification in Gauteng.

\section{Gender}

Analysis of sex ratios in NTDs showed an excess of affected female births, as expected from the literature. ${ }^{[6,9,11,13,28]}$ In addition, the correlation between the site of lesions and sex of the affected individuals was similar to that found in previous studies, with higher lesions occurring more often in females and lower lesions in males. ${ }^{[11,28]}$ Several theories have been postulated to explain these observations and include the suggestion that the process of neurulation may occur at slightly different times in the development of male and female embryos, thereby accounting for differing susceptibilities to the effects of factors such as teratogens. ${ }^{[11]}$ Other explanations, such as the involvement of $\mathrm{X}$ chromosome inactivation and maternal imprinting in the aetiology of NTDs, have also been postulated. ${ }^{[31]}$ The observation that neural tube closure occurs by a multisite process ${ }^{[2]}$ may also be involved if, for example, different sites are more susceptible to failure of closure in the different sexes. ${ }^{[32]}$

\section{Parental age}

Comparisons of maternal ages of women with offspring with NTDs showed no deviation from the distribution of maternal ages in the general population or from averages calculated from files of patients with disorders known to be unrelated to maternal age. This has been recorded in several other studies. ${ }^{[12,13]}$ Similarly, no paternal age effects could be demonstrated, a finding also documented in other studies. ${ }^{[6,14]}$

\section{Spontaneous abortions}

Mothers of individuals with NTDs had a significantly increased risk of spontaneous abortions as recorded previously. ${ }^{[15-17]}$ The increase in spontaneous abortions, despite the exclusion of pregnancies known to be affected by NTDs from the analysis, may be explained by the occurrence of NTDs or other congenital abnormalities in fetuses in which anomalies were present but not recognised, ${ }^{[15]}$ or may suggest that a history of miscarriage is an independent risk factor for NTDs.

\section{Maternal parity}

Significantly more NTDs occurred in firstborn offspring than in other offspring, 
indicating that there may be a parity effect on the aetiology of NTDs. This observation has also been made by others. ${ }^{[12,14]}$ Since no maternal age effects were demonstrated in the study, the observation is unlikely to be directly due to age-related factors. A possible contributing factor is that first pregnancies may be more likely to be unplanned and may be associated with more risk of maternal teratogen exposure and the effects of inadequate diet and nutritional deficiencies. Alternatively, an unfavourable pregnancy outcome in the first pregnancy may deter couples from planning further pregnancies. Fifth and higher pregnancies also showed an increased occurrence of NTDs. Larger families are a feature of lower socioeconomic groups and the inadequate spacing of pregnancies is often complicated by factors such as poor nutrition and cumulative nutrient deficiencies. A similar $\mathrm{U}$-shaped distribution for the occurrence of NTDs with parity was reported in a study performed in Cape Town. ${ }^{[9]}$

\section{NTDs and other malformations}

Individuals with NTDs were shown to have three times the postulated population risk of an unassociated congenital malformation (such as clefting), which is in keeping with other findings recorded in the literature. ${ }^{[1,1,19]}$ This may be due to the effects of teratogens or other factors on various areas of embryogenesis or to the occurrence of syndromic NTDs which were not diagnosed by the practitioner at the time the patient attended the genetic clinic. An example of such a condition is the autosomal recessively inherited Meckel syndrome in which NTDs, clefting and polydactyly may occur. ${ }^{[27]}$ Anti-epileptic medication may also be associated with NTDs and clefting. ${ }^{[27]}$ It was also shown that siblings of individuals with NTDs had a higher risk of congenital malformations other than NTDs (1/20 v. 1/30 postulated for the general population). When considering this finding, it is important to note that the actual background prevalence of birth defects is not known for this population and that data have been extrapolated from the literature from other populations. However, the findings of increased malformations in individuals with NTDs and in their siblings have relevance for the genetic counselling of, and the surveillance of pregnancies in, families affected by NTDs.

\section{Study limitations}

This retrospective study relied on the information (sometimes incomplete) available in the files of patients and was carried out on a relatively small sample several years ago, prior to the widespread implementation of dietary folate fortification. Further research would be worthwhile to determine the effects that this fortification has had on the occurrence and RRs for NTDs in the local population.

\section{Conclusions}

This study, as with studies performed in other populations, showed that RRs for NTDs tend to vary according to the population incidence of NTDs, with areas of lower incidence having lower rates of recurrence. ${ }^{[3]}$ An RR for NTDs after one affected child of $2.28 \%(1 / 45)$ and of $4.16 \%(1 / 24)$ after two affected children was calculated for the population. Analysis of the sex ratios among those with NTDs showed that significantly more females (M:F ratio 0.82) were affected. Several recent studies have suggested an appreciable decline in the incidence of NTDs at birth. This decline may be attributable to various factors including the increased use of prenatal diagnosis and selective termination of affected pregnancies in many parts of the world, better education and perinatal care facilities in many populations, as well as the increasing introduction of dietary folic acid fortification. ${ }^{[4,5,29]}$ It remains to be seen how occurrence and RRs of NTDs will change with the widespread fortification of food products with folic acid, with better education of mothers, and better antenatal healthcare services in our population. These findings have relevance to the counselling and care of family members of patients with NTDs.

Numerous risk factors have been documented in association with the occurrence of NTDs in various populations. This study showed that while maternal age was not a significant risk factor locally, maternal parity did play a role, with an increased risk documented in nulliparous and multiparous mothers. An increased occurrence of spontaneous abortions and unrelated congenital malformations were recorded in affected families.

NTDs remain a common cause of morbidity and mortality locally, and this study presents RRs and an understanding of the risk factors specific to the SA population, which will be useful in genetic counselling.

Acknowledgements. Contributors to this work included the late Sister Esther Zwane (who interviewed the subjects), Dr Tony Lane (statistical advice) and Professor T Jenkins (previously Head of Department). A scholarship from the Beit Trust and also a postgraduate merit award from the University of the Witwatersrand to GT, and the Mellon Mentorship Scheme support for JGRK, are gratefully acknowledged.

\section{References}

1. Kromberg JGR, Jenkins T. Common birth defects in South African Blacks. S Afr Med J 1982;62:599-602. 2. Carter CO, Evans K. Spina bifida and anencephalus in Greater London. J Med Genet 1973;10:209-234. Hall JG, Solehdin F. Genetics of neural tube defects. MRDD 1998-4(4) 269-281. [http://dx doiorg/10.1002/ (SIC) 1038-2779(1998) 1.4 269..AID-MRDD6>3.0.CO;2-8]

Frey L, Hauser WA. Epidemiology of neural tube defects. Epilepsia 2003;44(Suppl 3):4-13. [http://dx.doi org/10.1046/.1528-1157.44.s3.2.x

Sayed A, Bourne D, Pattinson R, et al. Decline in the prevalence of neural tube defects following folic acid fortification and its cost-benefit in South Africa. Birth Defects Research 2008;82(4):211-216. [http://dx.doi. org/10.1002/bdra.20442

Field B. Neural tube defects in New South Wales, Australia. J Med Genet 1978;15(5):329-338. [http://dx.doi org/10.1136/jmg.15.5.329]

Yen S, MacMahon B. Genetics of anencephaly and spina bifida? Lancet 1968:2(7568):623-626. [http:// dx.doi.org/10.1016/S0140-6736(68) $90709-5]$

Elwood JM, Little J, Elwood JH. Epidemiology and Control of Neural Tube Defects. New York: Oxford University Press, 1992.

Buccimazza SS, Molteno CD, Dunne TT, et al. Prevalence of neural tube defects in Cape Town, South Africa. Teratology 1994;50(3):194-199. [http://dx.doi.org/10.1002/tera.1420500304]

0. Lubinsky MS. Classifying sex biased congenital anomalies. Am J Med Genet 1997;69(3):225-228. [http:// dx.doi.org/10.1002/(SICI)1096-8628(19970331)69:3<225::AID-AJMG1>3.0.CO;2-K]

1. Seller MJ. Neural tube defects and sex ratios. Lancet 1986;328(8500):227. [http://dx.doi.org/10.1016/S01406736(86)92530-4

2. Williamson EM. Incidence and family aggregation of major congenital malformations of central nervous system. J Med Genet 1965;2(3):161-172. [http://dx.doi.org/10.1136/jmg.2.3.161]

3. Hunter AGW. Neural tube defects in Eastern Ontario and Western Quebec: Demography and family data Am J Med Genet 1984;19(1):45-63. [http://dx.doi.org/10.1002/ajmg.1320190108]

4. Carter CO, David PA, Laurence KM. A family study of major central nervous system malformations in South Wales. J Med Genet 1968;5(2):81-106. [http://dx.doi.org/10.1136/jmg.5.2.81]

15. Evans DR. Neural tube defects: Importance of a history of abortion in aetiology. BMJ 1979;1(6169):975-976 [http://dx.doi.org/10.1136/bmj.1.6169.975]

16. Myrianthopoulos NC, Melnick M. Studies in neural tube defects I. Epidemiologic and etiologic aspects. Am J Med Genet 1987;26(4):783-796. [http://dx.doi.org/10.1002/ajmg.1320260405]

17. Carmi R, Gohar J, Meizner I, et al. Spontaneous abortion - high risk factor for neural tube defects in subsequent pregnancy. Am J Med Genet 1994;51(2):93-97. [http://dx.doi.org/10.1002/ajmg.1320510203]

18. Creasy MR, Alberman ED. Congenital malformations of the central nervous system in spontaneous abortions. J Med Genet 1976;13(1):9-16. [http://dx.doi.org/10.1136/jmg.13.1.9]

19. Hall JG, Friedman JM, Kenna BA, et al. Clinical, genetic and epidemiological factors in neural tube defects. Am J Hum Genet 1988;43:827-837.

20. The Medical Task Force on Anencephaly. The infant with anencephaly. N Engl J Med 1990;322:669-674. [http://dx.doi.org/10.1056/NEJM199003083221006

21. David TJ, McCrae FC, Bound JP. Congenital malformations associated with anencephaly in the Flyde peninsula of Lancashire. J Med Genet 1983;20:338-341

22. Rodríuez JI, Rodríuez-Peralto JL, Muro M, et al. Anencephaly and limb deficiencies. Am J Med Genet 1992;44(1):66-71

23. MacMahon B, Pugh TF, Ingalls TH. Anencephalus, spina bifida and hydrocephalus: Incidence related to sex, race and season of birth, and incidence in siblings. J Epidemiol Community Health 1953;7:211-219. [http:// dx.doi.org/10.1136/jech.7.4.211]

24. Byrne J, Cama A, Vigliarolo M, et al. Patterns of inheritance in Irish and Italian families with neural tube defects: Comparison between high and low rate areas. Ir Med J 1997;90:32-34

25. Statistics South Africa. Census 1993. Pretoria: Central Statistic Service, 1993.

26. Hain FM. Aspects of twinning in selected South African population groups (MSc dissertation) Johannesburg: University of the Witwatersrand, 1993.

27. Harper PS. Practical Genetic Counselling. 7th Ed. London: Hodder Arnold, 2010.

28. Deak KL, Siegel DG, George TM, et al. Further evidence for maternal genetic effect and sex-influenced effect contributing to risk for human neural tube defects. Birth Defects Res 2008;82(10):662-669. [http:// dx.doi.org/10.1002/bdra.20511]

29. Grosse SD, Collins JS. Folic acid supplementation and neural tube defect recurrence prevention. Birth Defects Res 2007;79(11):737-742. [http://dx.doi.org/10.1002/bdra.20394]

30. Kromberg JGR, Wessels T-M, Krause A. Roles of genetic counsellors in South Africa. J Genet Counsel 2013 (in press).

1. Hall JG. Neural tube defects, sex ratios, and X inactivation. Lancet 1986;2(8519):1334-1335.

32. Seller MJ. Sex, neural tube defects, and multisite closure of the human neural tube. Am J Med Genet 1995;58(4):332-336. [http://dx.doi.org/10.1002/ajmg.1320580406]

Accepted 12 August 2013 